\title{
城市化进程对氮循环格局及动态的 影响研究进展
}

\author{
高 群 ${ }^{1}$, 余 成 ${ }^{1,2}$ \\ (1. 中国科学院流域地理学重点实验室,中国科学院南京地理与湖泊研究所,南京 210008 ; \\ 2. 中国科学院大学,北京 100049)
}

\begin{abstract}
摘 要: 氮循环是全球生物地球化学循环的重要组成部分, 随着城市化进程的不断加快, 高强度的人类活动和城市 景观格局的改变不仅影响了氮的循环过程, 而且加重了氮污染的程度,对城市生态环境造成了严重的影响。城市尺 度的氮循环研究不仅是全球关注的氮研究热点, 而且也是城市生态学和城市地理学研究的前沿。本文基于城市生 态系统的氮循环特征, 综述了城市化进程对城市氮污染物来源、沉降、输移和累积影响研究的国内外大量研究成 果, 介绍了卫星遥感、稳定同位素、物质流模型等分析方法在城市氮循环研究中的应用, 针对未来几十年活性氮增 加可能带来的不利影响, 提出对活性氮的减缓策略, 以有效抑制活性氮对环境的影响。未来城市氮循环研究需要 开展长期系统监测、深化城市氮循环机理研究、创新研究范式和研究方法, 并将研究成果与城市规划和城市发展研 究相结合,提升城市氮管理能力。
\end{abstract}

关 键 词: 城市雾霾;氮循环; 氮收支; 城市生态系统; 城市化

\section{1 引言}

氮循环涉及范围广、对生态环境的影响巨大， 是主要的生物地球化学循环之一, 也是全球关键生 物地球化学循环中受人类活动影响最大的(Galloway et al, 2004; 朱兆良等, 2010)。人类对全球氮循 环的影响既包括因氮肥使用导致粮食产量大幅度 的增加, 也包括对人类健康的影响、生物多样性丧 失和气候变化的代价(Erisman et al, 2013); 而从工 业和交通运输进人环境的活性氮(Reactive Nitrogen, $\mathrm{N}_{\mathrm{r}}$ ), 进一步扩大了这种影响的规模和范围 (Fowler et al, 2013)。据估算, 在 19 世纪 90 年代-20 世纪 90 年代的 100 年间, 全球人为活化氮的输人 量从 $15 \mathrm{Tg} / \mathrm{a}\left(1 \mathrm{Tg}=10^{6} \mathrm{t}\right)$ 增加到 $140 \mathrm{Tg} / \mathrm{a}$, 增长了将 近 10 倍, 并且这种趋势还在进一步加强(Galloway et al, 2002)。1910-2010年的近 100年间, 中国年均 活性氮的净产生量增加了 6 倍多。人为活动源首次
超过自然源出现在 1956 年, 到 2010 年其贡献达到 了 $80 \%$ 以上(Cui 等, 2013)。活性氮的污染对人类和 生态系统健康造成的影响包括饮用水(硝酸盐)、空 气质量(烟雾、颗粒物、地面臭氧)、淡水水体富营养 化、生物多样性丧失、平流层臭氧耗竭、气候变化和 沿海生态系统恶化等等(Erisman et al, 2013)。21世 纪面临的挑战为: 如何优化使用活性氮, 同时最大 限度地减少活性氮对人类和生态系统健康的负面 影响(Galloway et al, 2013)。

尽管城市及其郊区的面积仅占陆地面积的 $2 \%$, 但全球人口半数以上生活在城市, 城市化过程 带来的土地利用变化和环境污染是全球变化的重 要方面(Grimm, Faeth, et al, 2008; Grimm, Foster, et al, 2008),随着城市人口的快速增长, 作为资源集中 消费与废物集中释放的区域,城市化进程已成为改 变地区及全球氮循环的重要过程之一(Grimm et al, 2000; Svirejeva-Hopkins et al, 2011)。目前有关氮

收稿日期: 2014-10; 修订日期: 2015-05。

基金项目: 中国科学院海外科教基地建设计划项目(SAJC201319); 中国科学院南京地理与湖泊研究所“一三五”重点项目 (NIGLAS2012135006)。

作者简介: 高群(1972-),女,满族,新疆伊宁人,副研究员,研究方向为区域可持续发展、城市与区域规划,E-mail: qgao@niglas.ac.cn。 
循环机理、循环过程、循环通量测定、氮排放清单及 其空间分析、氮排放的环境影响等方面的文献很 多,但由于城市生态系统氮循环的空间复杂性和过 程动态性, 使得一些经验性的传统地理学和生态学 方法具有局限性, 人类活动对城市生态系统结构和 功能的控制作用和关系很难定量表征(Pickett et al, 2011), 城市尺度的氮循环研究仍然比较薄弱 (Grimm, Faeth, et al, 2008), 氮循环相关的城市环境 问题机理不明, 调控措施难以发挥作用, 还需要进 行更深人的研究。本文通过对近年来以城市为对 象的相关研究进行梳理, 基于氮素在城市特定的输 人输出迁移过程(Baker et al, 2001; Han et al, 2011) (图 1), 对城市氮的来源、氮素在城市中的迁移与转 化及其模拟与监测技术的研究进展等进行综述, 探 讨城市化过程对城市氮循环的影响, 深人研究城市 尺度氮循环变化及其生态效应, 提出该领域研究的 未来发展趋势, 旨在为实施新型城镇化规划进程 中, 协调好城镇化与生态环境的关系提供科学 依据。

\section{2 城市氮循环特征}

人类主导的城市生态系统有独特的氮收支项 目, 城市氮输人可分为自然输人和人为输人。自然 输人包括地表水输人、植物固氮和大气沉降; 人为 的输人主要是以供给人类消费为核心, 包括直接食 品输人、农作物生物固氮、工业产品和化石燃料燃 烧产生的氮氧化物等, 食物氮、能源氮和工业氮等 氮流构成其特殊的活性氮输人项目。氮素进人城
市后再经硝化、反硝化及氨挥发等过程以气态形式 返回大气,或以液态形式经地表径流和土壤淋失输 出到各类水体(Svirejeva-Hopkins et al, 2011), 部分 氮素在城市生态系统内存储和转型。Lin 等(2015) 总结了高密度、人类主导、低生态效用等城市氮循 环的特征发现, 强烈的人为作用是城市化地区氮循 环最突出的特点。研究表明, 城市中循环的活性氮 量已经超过周边非城市生态系统数倍(Jordan et al, 2003; Groffman et al, 2004; Kaushal et al, 2008)。城 市生活对粮食、工业品和能源相对较高的需求驱动 了农村地区高通量的食物氮生产, 以及工业固氮和 合成材料的加速发展,包括化石燃料中有机氮的大 量释放, 但人为输人的氮能被人类有效利用的部分 只占很小比例, 大部分都在环境中流失或累积(Erisman et al, 2013; 冼超凡等 2014)。Baker(2001)等在 亚利桑那州凤凰城城市生态系统的氮收支研究中 发现, 人为的氮输人占 $88 \%$, 其中食品和肥料的输 人占 $1 / 2$, 化石燃料燃烧的氮输人占 $1 / 3,21 \%$ 的氮在 城市生态系统内累积。累积下来的活性氮主要分 布在大气、土壤、河流水系和森林绿地等生态子系统 中。人类活动引起的氮输人和累积对区域环境特别 是水环境有着潜在的重要影响(Han et al, 2011)。

氮在城市生态系统中的循环过程不仅受城市 自然生态特征的影响, 而且氮素在城市中的输人、 扩散、转化、去除和累积,受城市发展进程的强烈扰 动(Jordan et $\mathrm{al}$, 1996), 其在城市各子系统及大气一 土壤一水体一生物各介质中的分布与流动规律十 分复杂。城市氮循环路径的改变主要受资源利用、 土地利用方式改变、废物产生与排放等人类活动影

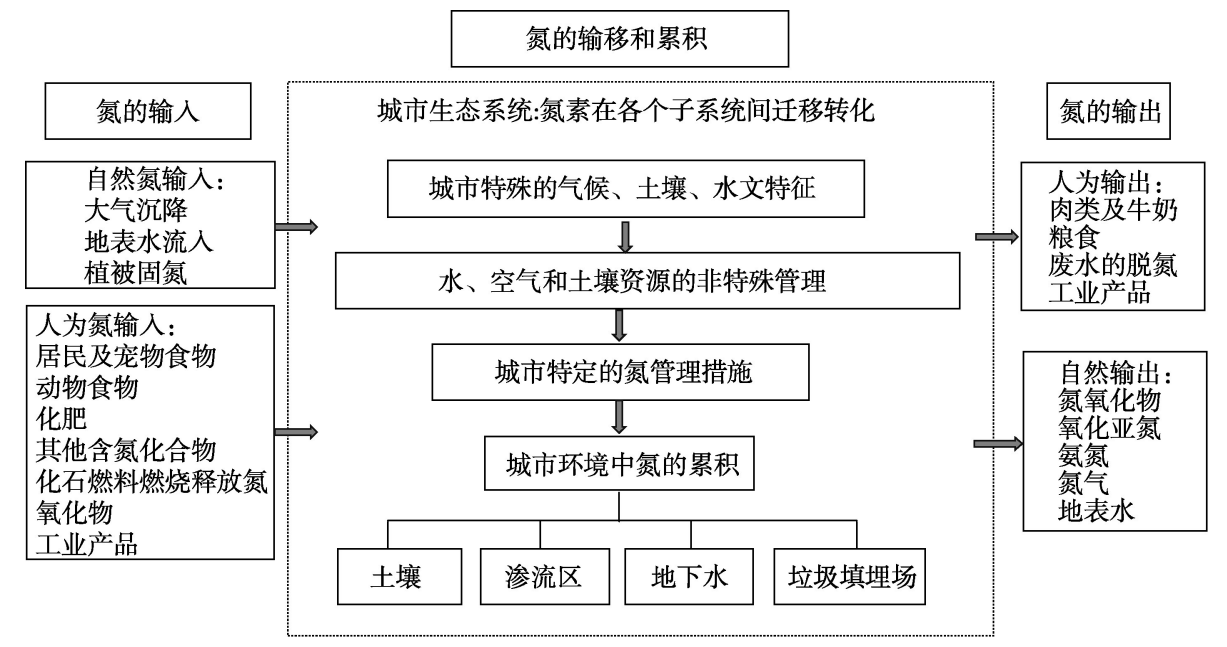

图 1 城市生态系统氮循环过程(Baker et al, 2001; Han et al, 2011)

Fig.1 Nitrogen circle in urban ecosystem(Baker et al, 2001; Han et al, 2011) 
响(Svirejeva-Hopkins et al, 2011), 如氮输人的形式 和数量、城市建设对自然水文循环的改变以及控制 氮污染的管理措施等的影响(Baker et al, 2001), 导致 氮素来源、量级、时空格局和驱动因素的改变 (Grimm, Foster, et al, 2008)。其中, 城市化扩张导致 的土地利用/土地覆盖快速变化对地表自然生态格 局和过程的显著改变是主要原因之一(Zhu et al, 2004; Burns et al, 2009)。近年来, Han 等(2011)对北 京市净人类活动氮输人和累积的分布情况以及与 人口分布及土地利用类型分布的关系研究, $\mathrm{Gu}$ 等 (2012)借助人与自然耦合系统法测定长期的城市化 如何影响上海地区的氮流、排放及其驱动因子的研 究, 以及高伟等(2014)采用本土参数对中国 358 个 城市地区人类活动净氮输人量的核算研究等, 加深 了对城市生态系统氮循环的认识。通过对城市尺 度氮循环变化全面的定性与定量分析, 有助于理解 城市化、经济发展等人为因素对活性氮通量变化的 影响。城市环境问题的生态学实质是物质与能量 代谢在时间和空间尺度上的滞留, 从而导致环境污 染与有害物质富集。当前, 探究人类活动如何影响 城市中的氮输人的空间格局? 城市生态系统如何 响应人类对氮循环的改变? 不同社会经济水平、政 策和行为特征如何影响城市氮代谢的方式、途径与 效率? 上述研究不仅是全球关注的氮研究热点, 而 且也是城市生态学和城市地理学研究的前沿。

\section{3 城市发展对氮循环关键过程的影响}

快速城市化进程中的城市人口增长、经济发 展、城市空间的快速扩张以及相应的政策调整, 强 烈地影响氮分布格局和动力学特征(Grimm, Faeth, et al, 2008), 主要表现为氮输人的增长。在时间尺度 上, 城市化发展的不同阶段, 城市的氮收支具有不 同的特征, 活性氮的输人开始从农业源占主导转向 工业和城市交通占主导(Fowler et al, 2013), 氮输人 与 GDP 增长之间存在显著的正相关。在空间尺度 上, 受城市化的影响, 郊区、郊区化地区、老住宅区、 城市核心区等不同城市功能区的氮输人、输出及持 留具有明显的空间差异(Groffman et al, 2004)。城 市空间结构类型 (紧凑或松散)直接影响城市的氮 流动(Svirejeva-Hopkins et al, 2011)。

\section{1 城市经济社会发展对城市氮源输入的影响}

城市的氮来源复杂, 高密度人口、密集的工业
布局和繁忙的城市交通导致排放到城市环境中的 活性氮量级急剧增长(Vitousek et al, 1997; 遆超普 等, 2010)。Howarth 等(1996)首次提出了净人类活 动氮输人(Net Anthropogenic Nitrogen Input, NANI) 概念, 以核算流域人类活动控制的氮源输人强度和 表征人类活动对区域氮循环的影响。许多流域尺 度的研究表明(张柏发等, 2014; 张汪寿等, 2014), NANI 在流域尺度对氮通量的影响更为敏感, 即使 其微小的变化也可能引起河流氮含量的较大变 化。高伟等(2014)对城市区域的研究表明,中国城 市净人类活动氮输人强度是全球平均水平的 3 倍; 在氮源结构上, 氮肥施用占输人总量的 $65.0 \%$, 其他 依次为食品/饲料净输人 $(21.4 \%)$ 、大气沉降 $(7.3 \%)$ 和 作物固氮 $(6.5 \%)$ 。目前, 国内外有关氮源、氮收支的 研究工作比较活跃, 主要通过建立排放清单和物质 流方法分析人为氮源输人数量及其分布特征, 但现 有的研究大多集中在较大区域尺度(如全国或省级) (施亚岗等, 2014)。氮源排放的重点区域难以在大 尺度上准确识别, 且大尺度的研究也难以为后续的 研究提供高精度的数据支撑。因此, 以更加精细的 城市空间尺度研究净人类活动氮输人成为国外内 研究的新趋势。

城市工业、电力企业和机动车尾气等由化石燃 料燃烧产生的温室气体占人为氮化合物输人的较 大份额(Butler et al, 2005; Elliott et al, 2007), 主要通 过大气沉降进人生态系统。除电力企业外, 钢铁、 水泥、石化等重化工业，虽通过改进生产工艺实行 减排,但因其产量巨大, 现仍是大气氮污染的重要 来源(薛亦峰等, 2014)。来自工业生产方面的输人 除化石燃料燃烧外, 还包括含氮工业产品(图 2), 总 体在城市氮通量中占较大比重(Galloway et al, 2008; 谷保静等, 2013), 加上服装、装饰品、药品、塑 料和建筑材料等在城市生态系统中较难分解的工 业含氮产品成为快速城市化地区城市氮代谢中的 主要氮流(冼超凡等, 2014)。

根据国内外多城市大气 PM2.5 源解析结果和 城市道路运输氮氧化物排放的监测结果(Beevers et al, 2012; Leach et al, 2012), 机动车尾气中的氮氧化 物气态污染物是主要来源并占较大比例。随着城 市化的不断推进, 城市交通流量不断增大, 交通产 生的氮氧化物已经远高于电力等工业企业的排放 量。风速、风向、环境温度、污染源、城市地形以及 城市居民通勤规律等因素通过影响道路交通氮污 


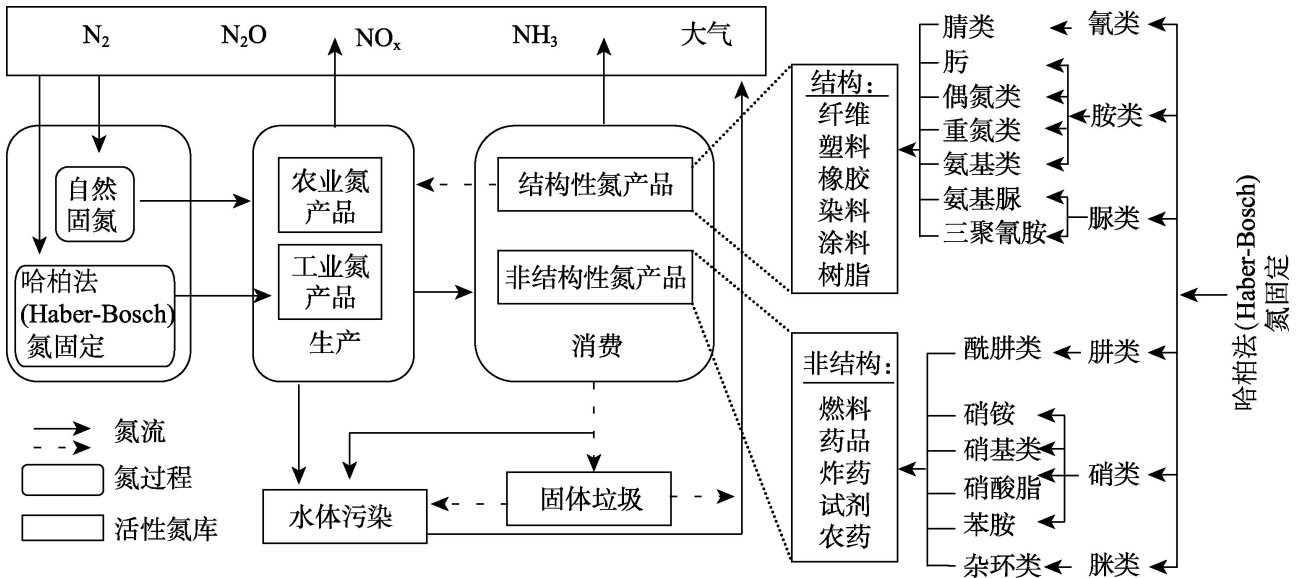

图 2 工业氮源循环过程(谷保静等, 2013)

Fig.2 Industrial $\mathrm{N}_{\mathrm{r}}$ sources and circle(Gu et al, 2013)

染物排放浓度和扩散, 致使城市中氮氧化物浓度存 在空间梯度差异和周循环现象等时空变化规律(申 卫国等, 2010; Cyrys et al, 2012)。

此外, 城市化的快速发展还导致城市生活污 水、生活垃圾、宠物粪便及城市住宅区或商业区绿 地的施肥等氮源输人的快速增长 (Burns et al, 2009), 和城市人口增长带来的对食物氮素需求。 生活水平提高导致饮食结构中植物性氮消费量和 动物性氮消费量不断增长, 由此导致城市居民产生 的粪尿和垃圾中氮排放量也大幅度增长(魏静等, 2008)(图 3)。城市食物氮代谢和氮足迹研究表明, 在垃圾和污水处理系统不完善的条件下,食物氮流 失到环境中去的比例较高(Forkes, 2007), 由于快速 城市化和经济发展带来的动物性食品比重大幅提 高, 导致食物氮足迹增加(秦树平等, 2011)。另外, 城市景观中肥料使用导致的 $\mathrm{N}_{2} \mathrm{O}$ 等温室气体的增
加往往被低估(Townsend-small et al, 2011), 由于城 市绿地和景观的高施肥率, $\mathrm{N}_{2} \mathrm{O}$ 年通量甚至大于农 业用地。

\section{2 城市不同下垫面对氮沉降的影响}

城市产生的氮氧化物和氨排放量的一半以上 又沉降回城市区域内(Russell et al, 1993), 成为城市 生态系统营养盐的重要来源(Sylvia et al, 2009)。高 氮沉降热点区主要位于城镇密集的城市群地区或 者大都市中心区(Fenn et al, 2003), 在京津冀、长三 角和珠三角等城市群地区(遆超普等, 2010), 以及上 海、南京、重庆、北京、大连、乌鲁木齐等大城市地区 都观测到较高的氮沉降通量。

大气氮沉降的时空差异除与氮排放和降雨量 等气象条件直接相关外(袁玲等, 2009), 沉降的速率 和通量在空间上受到城市不同下垫面的支配,包括 城市的建筑格局以及树木的种类和分布等(Rojas

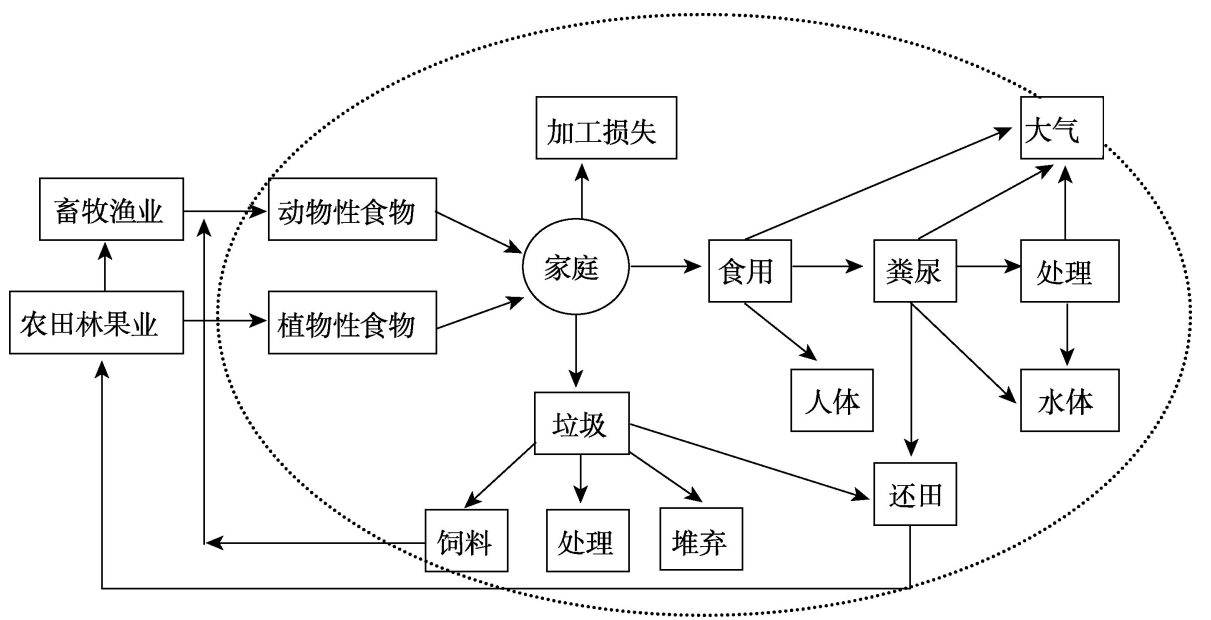

图 3 食物氮源流动模式(魏静等, 2008)

Fig.3 The nitrogen flow model in food sources(Wei et al, 2008) 
et al, 2009), 具有明显的空间异质性。城市下垫面 的变化, 如城市建筑物密集程度增加, 林地、草地、 水面等生态开敞空间的减少, 导致城市风速、温度 等小气候条件发生变化,城市生态系统对污染物自 净能力下降, 进而影响氮污染物的吸收和扩散, 尤 其是对氮干沉降影响更明显。大气干湿沉降通量 与氮通量观测点的位置和土地利用特征之间存在 相关关系(Luo et al, 2003), 从城市中心到乡村一般 呈梯度下降趋势 (Lohse et al, 2008; 邓君俊等, 2009)。农业区氮沉降通量高于非农业区, 城市地 区氮沉降通量高于森林地区(Bettez et al, 2013), 而 受工业布局和交通干线影响的大气硝态氮和亚硝 态氮沉降往往具有线源和点源特征(王德宣等, 2010); 与运输相关的氮气溶胶或 NOx 沉降高值接 近公路, 氨含量高值则可能接近饲养场或城乡结合 部的农业区(Elliott et al, 2007)。城市工业集中区、 商业区和居住区等功能板块高度集中分布, 各不同 功能区降水径流中氮元素均具有较高的含量水平, 其中工业区和交通要道降水径流氮含量比居民居 住区、商业区和休㽞区域普遍偏高(蒋海燕等, 2002; 夏立忠等, 2003), 有的居民区分布在工业区 的下风向, 也导致氮污染物的叠加复合。

\section{3 城市不同扩张方式对氮流动的影响}

在城市生态系统复杂条件下, 多介质多界面环 境中氮素的输移和累积是城市氮循环的重要过程 (Flint et al, 2007), 也是城市生态学相关研究的热点 和难点。过去 30 年中, 大量的活性氮通过自然和人 为固氮方式已在城市的植物、土壤和水体中累积 (图 1), 氮素的输移和累积对城市水体和空气质量有 重要影响 (Jansson et al, 2007; Schlesinger, 2009; Han et al, 2011)。城市化进程不断侵占森林、农田、 湿地等生态空间, 导致城市不透水覆盖范围扩大、 植被减少、河流渠道化、湿地退化、河岸带退化等, 对 氮素在城市的输移和累积产生巨大干扰(Ortiz-Zayas et al, 2006; Kaushal et al, 2008)。Groffman 等 (2004)发现, 草坪、河岸带和湿地等为城市中重要的 氮持留地区, 对城市氮循环有重要调节功能, 是削 减进人河流 $\mathrm{NO}_{3}^{-}$的有效 “汇” (Groffman et al, 2004)。湿地的反硝化作用有利于城市非点源氮污 染的削减(Zhu et al, 2004), 是截留转化陆地污染物, 防止其进人城市水体的重要场所(娄焕杰等, 2013)。但目前对于城市地区氮持留还缺乏机理方 面的研究, 相关研究结论存在较大经验性, 氮持留
比例变化区间较大。例如 Whitall 等(2001)估计在 城市流域的氮持留率为 $25 \%$ 95\%; Wollheim 等 (2005)发现在马萨诸塞州的城市流域有 $65 \%$ 85\% 的氮持留,而湿润年低于干燥年;Kaushal 等(2008) 也发现在巴尔的摩郊区流域,气候差异影响着氮在 城市的持留, 从干燥年的 $85 \%$ 下降到湿润年的 $35 \%$ 。

在城市暴雨的情况下, 如果没有任何干预性持 留措施, 相比周边未开发的生态系统, 由于城市化 进程通过增加不透水地面的覆盖范围和改变供排 水设施, 降低降水的土壤入渗从而增加地表径流, 改变了城市生态系统中自然的水文过程。降雨在 城市生态系统中产生更大的径流量、更高的峰值流 量和更急剧的水文过程(Paul et al, 2001; 李恒鹏等, 2006), 城市表面积累的氮通过地表径流直接运送 到城市水体。Roberts 等 $(2009,2010)$ 通过 SPARROW 模型模拟发现, 不同的城市化模式对氮输移 的影响存在较大差异: 以低密度分散的住宅和商业 发展为主的城市化模式,城市不透水地面的扩大并 未导致进人水体总氮水平的大幅提高; 而高密度连 片发展的城市化模式, 导致摊大饼式的城市发展形 态,则会提高非点源氮的输出量。

\section{4 城市氮循环的监测、模拟与分析方法}

受城市氮循环过程复杂性和尺度差异的影响, 在城市进行准确的动态监测和定量研究具有较大 的难度。国外也仅有凤凰城、巴尔的摩等少数城市 正在开展较长期的氮循环观测和研究(表 1), 大多数 相关研究监测样点较少、研究周期较短, 导致研究 结果存在较大的不确定性。国内外在微观尺度氮 循环研究中已提出了很多机理模型, 但是在大、中 尺度城市区域的氮循环研究中, 由于人类主导的生 态系统的氮循环规律复杂性, 主要依靠经验、半经 验模型进行模拟或估算。已建立的用于农业、森林 及土壤等生态系统的物质循环模型并不适用于城 市系统。传统的测量活性氮浓度和通量的方法在 时间和空间的应用方面受到限制, 而模型模拟通常 需要浓度测量的验证。目前在城市地区, 利用卫星 遥感技术进行空间差异分析、运用物质流分析模型 进行城市氮循环各关键过程的模拟，以及稳定氮同 位素 $\left(\delta^{15} \mathrm{~N}\right)$ 分析技术对氮污染监测与示踪, 可对污 染物的来源以及在城市生态系统内的流动与循环 
表 121 世纪以来国内外城市尺度城市发展的氮循环影响研究工作

Tab.1 Global studies that focus on nitrogen circle of urban scale since the 21 st century

\begin{tabular}{|c|c|c|c|}
\hline 研究区域 & 研究内容 & 研究方法 & 文献来源 \\
\hline 美国亚利桑那凤凰城(CAP) & 城市生态系统氮收支平衡 & 质量平衡法 & Baker et al, 2001 \\
\hline 美国马里兰巴尔的摩市(LTER) & 城市流域生态系统氮通量和持留 & 质量平衡法 & Groffman et al, 2004 \\
\hline 瑞典斯德哥尔摩市 & $\begin{array}{l}\text { 基于模拟不同城市发展情景产生的氮负荷提出城 } \\
\text { 市空间扩展方向 }\end{array}$ & 水文学模型模拟 & Jansson et al, 2007 \\
\hline 法国巴黎市 & 氮流在城市食物消费中的流动情况 & 物质流分析 & Barles, 2007 \\
\hline 加拿大多伦多市 & 氮流在城市食物消费中的流动情况 & 物质流分析 & Forkes, 2007 \\
\hline 美国亚利桑那凤凰城(CAP) & 城市大气氮沉降空间格局特征 & 干湿沉降采样及统计分析 & Lohse et al, 2008 \\
\hline 中国惠州市 & $\begin{array}{l}\text { 与人类活动有关的氮流在环境系统和人类生产生 } \\
\text { 活系统的状况 }\end{array}$ & 物质流分析 & Ma et al, 2008 \\
\hline 中国贵阳市 & 城市大气氮沉降空间格局特征 & 氮稳定同位素分析 & 刘学炎等, 2009 \\
\hline 中国大杭州区域(GHA) & 城乡复杂系统内氮循环状况 & 物质流分析 & Gu et al, 2009 \\
\hline 中国南昌市 & 城市化对森林氮循环的影响 & 离子树脂采样及统计分析 & Chen et al, 2010 \\
\hline 中国北京都市区 & 城市地区净人类活动氮累积研究 & 氮输人输出清单 & Han et al, 2011 \\
\hline 中国大上海地区(GSA) & 城市化对城乡区域氮流动及其驱动力的影响 & 物质流分析 & Gu et al, 2012 \\
\hline 美国佛罗里达彭萨科拉市 & 城市景观特征影响河口氮负荷 & 景观指数分析 & Yang, 2012 \\
\hline 中国厦门市 & 城市发展过程中食物氮消费的变化 & 物质流分析 & 于洋等, 2012 \\
\hline 中国北京市 & 城市化对土壤氮循环的影响 & 土壤理化性质分析 & 陈帅等, 2012 \\
\hline 中国北京都市区 & 城市生态系统地表水氮污染和来源识别 & 氮稳定同位素分析 & Ren et al, 2014 \\
\hline 中国北京都市区 & $\begin{array}{l}\text { 大都市地区的快速增长与在食品生产消费体系中 } \\
\text { 氮磷的流动变化关系 }\end{array}$ & 物质流分析 & Ma et al, 2014 \\
\hline 巴西里约热内卢市 & 大城市发展对相邻地区生态系统氮循环的影响 & 降雨采样及统计分析 & De Souza et al, 2015 \\
\hline
\end{tabular}

进行综合定量研究。

\section{1 利用卫星遥感方法监测城市地区氮氧化物排放} 情况

利用遥感技术进行环境监测可获得常规地面 观测手段无法获取的重要参数, 利用臭氧探测仪 (Ozone Monitoring Instrument, OMI) 等多种卫星传 感器观测到的对流层 $\mathrm{NO}_{2}$ 浓度能与地面排放的 NOx 建立直接的联系, 反映出污染地区化石能源消 耗和污染物排放的强度(Akimoto et al, 2006; Zhang et al, 2007)。根据卫星获得的对流层 $\mathrm{NO}_{2}$ 柱浓度数 据, 北京到上海之间的工业与城市密集地区已成为 世界上对流层 $\mathrm{NO}_{2}$ 污染最为严重的地区(张强等, 2012)。而 Beirle 等 (2003) 应用 GOME(Global Ozone Monitoring Experiment)利用观测到的 $\mathrm{NO}_{2}$ 柱 浓度数据对全球工业化和城市氮氧化物排放的周 循环现象进行评估; 余环等(2009)利用美国 Aura卫 星装载的 $\mathrm{OMI}$ 仪器观测反演得到的对流层 $\mathrm{NO}_{2}$ 柱 浓度资料, 通过与同期北京及周边城市天津和唐山 的综合比较,评估了北京奥运期间空气质量保障措 施的实施效果。Lamsal 等(2013)利用 OMI 反演的
$\mathrm{NO}_{2}$ 柱浓度数据, 分析全球城市人口集聚与氮氧化 物排放的关系, 指出 $\mathrm{NO}_{2}$ 浓度水平受工业化和人均 排放量的影响,并存在明显的区域差异。

\section{2 利用稳定氮同位素分析方法识别和追踪氮源}

稳定氮同位素 $\left(\delta^{15} \mathrm{~N}\right)$ 分析技术近年来开始用于 人为活动干扰强烈的城市生态系统氮循环过程研 究。与传统的氮浓度和通量的测量方法以及氮循 环模型模拟方法相比, 稳定同位素研究在识别和追 踪氮循环中氮素的人为和天然来源方面提供了新 的视角,携带不同稳定同位素特征的氮源可以用来 评估不同来源氮通量是如何变化的(Hastings et al, 2013)。作为一种示踪手段, 可以根据不同形态 $\left(\mathrm{NH}_{4}{ }^{+} 、 \mathrm{NO}_{3}{ }^{-}\right)$的氮稳定同位素比值的差异进行氮污 染源的识别以及对城市氮循环变化进行综合研究 (林光辉, 2013; Felix et al, 2014; 徐志伟等, 2014)。 例如 Elliott等(2007)利用氮同位素在美国东北部进 行硝酸盐沉降研究, 揭示了硝酸盐 $\delta^{15} \mathrm{~N}$ 与电厂前体 物 NOx 排放之间的相关关系; 刘学炎等(2009)通过 石生苔藓氮含量和氮同位素组成分析研究贵阳城 区和乡村地区氮污染物来源和浓度的空间差异性; 
岳甫均等(2010)分析天津市地表水体中硝态氮和氨 氮的 $\mathrm{N}$ 同位素组成结合研究水体的水化学特征, 识 别出地表水体中氮主要来源为生活污水, 而且氮形 态转化受硝化作用、挥发作用以及可能的反硝化作 用影响明显。特别是 $\delta^{15} \mathrm{~N}$ 和 $\delta^{18} \mathrm{O}$ 双同位素技术能更 准确地示踪氮污染源和了解氮循环过程(Townsendsmall et al, 2011)。Ren等(2014)对北京市城区内水 体 $\mathrm{NO}_{3}$ 的同位素示踪研究表明, 上游土地利用方式 主要为公园和绿地, 水体 $\mathrm{NO}_{3}$ 主要来自土壤有机氮 及大气氮沉降, 而下游随着居住用地及商业用地面 积增加, $\mathrm{NO}_{3}$ 污染来源则主要为居民生活污水及工 业废水。

\section{3 利用物质流方法分析城市氮代谢路径}

在城市生态系统中, 人类既是系统的参与者又 是调控者, 使得传统的生态系统模型难以直接用于 人类主导的系统(Kaye et al, 2006)。从综合视角评 估区域性氮收支(Nitrogen Budget)与氮平衡(Nitrogen Balance) 是科学地阐述一个地区氮循环进而掌 握氮污染的主要来源和途径, 并对该地区氮素进行 科学管理的关键(Xing et al, 2002; 郑循华等, 2002; 邓美华等, 2007)。物质流分析方法(Material Flow Analysis)采用测量、估算、文献评述、专家评估的方 式, 通过分析国内外权威机构的报告、政府统计数 据、正式发表的文献以及卫星遥感数据等, 量化输 人、输出人类主导的城市生态系统的氮流及氮在系 统内部的格局与过程, 确定物质的源、汇和传输途 径(Ma et al, 2008), 已被用于多个城市尺度区域的 氮循环分析。该方法主要以质量平衡(Mass balance)理论(Kaye et al, 2006)为基础, 以“大城市地区 (Great Urban Area)”城市一乡村的复合生态系统为 研究对象, 分多个子系统进行氮的输人、内部转化 与输出分析, 根据氮在各子系统间的转化过程, 建 立城市复合生态系统中各形态氮化合物迁移转化 模型, 从而判断一个城市是氮源(输人 > 输出)或氮 汇(输人<输出), 发现城市氮循环的规律。例如, Baker等(2001)将亚利桑那州凤凰城复合生态系统 分为地表水子系统、近地表大气子系统、沙漠子系 统、农田子系统、养殖产业子系统、人类食物需求子 系统、宠物子系统、废水子系统、城市景观子系统以 及地下子系统; $\mathrm{Gu}$ 等(2009)将大杭州地区分为生产 者、消费者、分解者和生命支持 4 个功能组团, 每个 功能组团包含一个或多个子系统。物质流分析需 要在氮收支计算中引人各个因子的多年数据, 比较
详尽的信息数据支持,对于基础信息不全的地区其 估算可能会出现偏差(Gu et al, 2012), 主要限制性 因素是城市系统边界的确定和不同城市核算结果 的比较。

\section{5 城市氮素循环失衡产生的环境问题 及对策}

氮素作为一种重要的营养元素, 是陆地生态系 统功能和过程的重要限制性因子, 也是非常难以控 制的污染物之一(Vitousek et al, 1997)。人类对自然 氮循环的干扰会导致环境及生态系统产生一系列 的氮连锁效应 (Nitrogen Cascade) (Galloway et al, 2013)。大量氮素的输人一旦超过城市生态系统的 承载能力将会带来严重的负面环境影响, 尤其是大 气中的 NOx 和水体中的活性氮引起的生态和环境 问题对人类的健康生存造成威胁。以大气环境为 例, 2012 年以来, 中国中东部大城市连绵区灰霧天 气频发, 北京城区 PM2.5 值一度逼近 1000 , 并检出 大量危险的含氮有机颗粒物。灰霾天气期间大气 $\mathrm{PM} 2.5$ 的主要组成成分是 $\mathrm{TC} 、 \mathrm{SO}_{4}{ }^{2-} 、 \mathrm{NO}_{3}{ }^{-}$和 $\mathrm{NH}_{4}{ }^{+}$, 王 占山(2014)等对北京地区 PM2.5 的时空分布研究表 明, $\mathrm{NO}_{2}$ 对 PM2.5 浓度的影响比 $\mathrm{SO}_{2}$ 更加显著。合 成纤维、塑料、医药等工业氮产品由于复杂的组成 和分解过程也会造成大量的固体废弃物污染, 许多 大城市陷人 “垃圾围城”的困境中, 其积累过程带来 的滞留效应对环境和人类健康存在长期的影响 $(\mathrm{Gu}$ et al, 2013; 谷保静等, 2013)。氮的污染已超越了人 类和城市生态系统健康的许多临界值(Erisman et al, 2013)。究其原因,城市人口的增加、工业的集 聚、自然资源的大量利用所造成的集中、高强度的 氮污染物输人无疑是主要原因,但中国大城市空间 格局不合理也是雾䨪等“城市病” 区域性爆发不能 忽视的重要根源之一(高吉喜等, 2014)。

城市的氮循环过程中存在复杂的相互作用, 而 且这些循环过程在时空尺度上存在着高度变异性, 不仅使人们对于城市氮循环问题难以充分了解, 而 且还使得制定有效的治理对策变得更为困难。在 2004 年举行的第三次国际氮素大会上,来自全球的 生态学家共同签署了《南京宣言》, 首次倡导在全球 范围内采用人为干预措施来缓解人类源活化氮带 来的生态系统与人类健康问题(Erisman, 2004)。城 市是氮污染物的源、汇或转换器 (Keeney et al, 
2008), 城市生态系统对于氮污染物的净化能力不 足是氮循环失衡的主要限制因素(Gu et al, 2012), 因此,通过对活性氮的来源和扩散途径进行控制可 以有效地抑制过量活性氮对环境的影响。为此,一 方面, 要从源头控制达到氮污染物减排的目的。城 市复合生态系统的氮循环受到人类活动的影响,也 意味着城市应该加大对氮污染物的管理和调控力 度,可通过交通、工业和农业部门共同采取措施达 到氮污染物减排目的。因此, 合理进行城市规划, 从人口与产业布局、公共交通等基础设施的合理建 设对氮污染起到调控作用。许多欧洲国家通过设 置城市环保区、覆盖粪便储存设施、发展低排放住 房等措施减少氮污染物的产生 (Erisman et al, 2003); 其中, 通过减少交通流量、汽车技术改进等 管理措施减少道路交通的氮氧化物排放是降低城 市氮输人最容易达成的途径之一(Carslaw et al, 2001)。另一方面, 要从氮素的输移和持留循环过 程中控制氮污染物的影响。通过将工程措施、生物 措施和蓄水保土相结合, 如生物滞留池、可渗透路 面、绿色屋顶、河岸植被缓冲带以及城市湿地等雨 水控制措施(Stormwater Control Measures, SCMS), 来滞留径流、增加人渗, 不仅可减轻城市洪峰流量 和减少城市内涝, 而且还可有效控制氮污染。通过 氮吸收和反硝化作用等脱氮机制实施氮污染物削 减功能(Kim et al, 2003; Hsieh et al, 2007), 可促使城 市雨水径流和城市不透水区域排水中的氮负荷减 少, 保护城市水体生态系统(Walsh et al, 2009; Larson et al, 2012)。

\section{6 研究展望}

城市生态系统是气候、土壤、植物和人类活动 等要素组成的有机整体, 各城市氮循环涉及地球多 圈层间的复杂过程和许多非线性关系, 城市化进程 中人类活动对氮循环过程的影响并非一个简单的 单向反馈过程, 而是一个极其复杂的动态过程, 仍 然存在一些不明确的响应关系, 需要综合考虑多方 面因素, 宏观定性分析与微观定量模拟、预测相结 合, 开展深人的机理分析和理论实践相结合的研 究。在城市尺度上把握氮素输人输出的变化规律、 氮污染的发展趋势及其驱动机制, 深人探讨城市氮 排放、氮沉降及氮收支平衡对城市生态系统的响应和 反馈,对合理制定政策措施和确保城市生态系统的
可持续发展具有重要意义, 是氮素污染控制领域的关 键科学问题。今后可在以下几方面作深人的研究:

(1) 选择典型城市建立快速城市化地区长期、 多点、全面的氮循环监测网络, 获得长期的观测数 据, 才能更好地认识城市氮循环机理, 分析氮循环 的水文和生物地球化学驱动因素以及氮循环对气 候变化和城市化进程复杂的响应特征(Collins et al, 2010), 为研究城市生态系统对氮循环变化的响应 和反馈提供必要的数据支持。

(2) 在实施新型城镇化规划和中国城镇化快速 发展的宏观背景下,城市化及其规划是将氮管理与 城市发展相整合的关键过程,未来几十年城市规划 和管理将对氮循环产生巨大影响。根据城市化对 氮循环的长期影响, 通过城市土地利用变化的氮循 环效应研究,结合生态、经济模型,分析不同的城市 发展格局(紧凑型或松散型)如何影响氮循环过程并 最终影响城市及其周边的生态系统, 回答如何将城 市化对氮循环模式及动力机制产生的负面影响降 至最低。要解决氮污染引起的城市生态环境问题, 既需要从控制污染物排放着手, 也需要从城市规 划、城市生态等多角度进行综合研究。

(3) 从城市氮代谢人手, 研究城市中氮代谢的 总量、效率、特征、结构、演变规律和趋势等, 是有助 于解决城市生态环境问题的一个新方向。通过阐 明城市化进程中社会经济水平、政策和行为方式对 城市氮代谢过程的影响,研究其动态作用过程及机 制, 有助于探索城市氮污染的优化控制模式和制定 有效的定向调控手段(洗超凡等, 2014)。

(4) 未来城市尺度氮循环研究应注重多学科交 叉, 宏观定性分析与微观定量模拟、预测相结合, 加 强研究人为活动干预下生态系统氮素生物地球化 学过程及其环境影响研究,加强城市生态系统中各 个要素之间的耦合研究, 将污染源数据和不同土地 利用类型的氮负荷数据相联系,将氮素流动的空间 特性和空间过程相结合(Burian et al, 2001; Jansson et al, 2007), 加强多学科间的交流与合作。基于城 市时空发展趋势, 未来应利用卫星遥感及稳定同位 素分析等技术手段,深人研究城市环境中人类活动 和大气、陆地和水体氮通量之间的动态耦合关系。

\section{参考文献(References)}

陈帅, 王效科, 逯非, 等. 2012. 城市化对城区和郊区森林土 壤氮循环的影响 [J]. 土壤通报, 43(4): 842-848. [Chen S, 
Wang X K, Lu F, et al. 2012. Effects of urbanization on soil nitrogen cycle in urban and suburban forests[J]. Chinese Journal of Soil Science, 43(4): 842-848.]

邓君俊, 王体健, 李树, 等. 2009. 南京郊区大气氮化物浓度 和氮沉降通量的研究 [J]. 气象科学, 29(1): 25-30. [Deng J J, Wang T J, Li S, et al. 2009. Study on atmospheric nitrogen oxidant and deposition flux in suburban of Nanjing [J]. Scientia Meteorologica Sinica, 29(1): 25-30.]

邓美华, 谢迎新, 熊正琴, 等. 2007. 长江三角洲氮收支的估 算及其环境影响 $[\mathrm{J}]$. 环境科学学报, 27(10): 1709-1716.

[Deng M H, Xie Y X, Xiong Z Q, et al. 2007. Nitrogen budgets of the Yangtze Delta region and their effect on the environment[J]. Acta Scientiae Circumstantiae, 27 (10): 1709 -1716. ]

高吉喜, 张惠远. 2014. 构建城市生态安全格局从源头防控 区域大气污染 [J]. 环境保护, 42(6): 20-22.[Gao J X, Zhang H Y. 2014. Goujian chengshi shengtai anquan geju cong yuantou fangkong quyu daqi wuran[J]. Environmental Protection, 42(6): 20-22. ]

高伟, 郭怀成, 后希康. 2014. 中国大陆市域人类活动净氮输 人量(NANI)评估[J]. 北京大学学报: 自然科学版, 50(5): 951-959.[Gao W, Guo H C, Hou X K. 2014. Evaluating city- scale net anthropogenic nitrogen input (NANI) in Mainland China[J]. Acta Scientiarum Naturalium Universitatis Pekinensis, 50(5): 951-959. ]

谷保静, 杨国福, 罗卫东, 等. 2013. 中国工业氮通量快速增 长的驱动力及其影响 $[\mathrm{J}]$. 中国科学: 地球科学. 43(3): 469-477. [Gu B J, Yang G F, Luo W D, et al. 2013. Rapid growth of industrial nitrogen fluxes in China: driving forces and consequences[J]. Science China: Earth Sciences, 56: 662-670 ]

蒋海燕, 刘敏, 顾琦, 等. 2002. 上海城市降水径流营养盐氮 负荷及空间分布 $[\mathrm{J}]$. 城市环境与城市生态, 15(1): 1517. [Jiang H Y, Liu M, Gu Q, et al. 2002. Nutrient nitrogen loading and spatial distribution of storm-water runoff in Shanghai City[J]. Urban Environment \& Urban Ecology, 15(1): 15-17. ]

李恒鹏, 黄文钰, 杨桂山, 等. 2006. 太湖上游典型城镇地表 径流面源污染特征[J]. 农业环境科学学报, 25(6): 15981602. [Li H P, Huang W Y, Yang G S, et al. 2006. Nonpoint source pollutant concentration in typical towns of Taihu upriver region[J]. Journal of Agro- Environment Science, 25(6): 1598-1602. ]

林光辉. 2013. 稳定同位素生态学 [M]. 北京: 高等教育出版 社. [Lin G H. 2013. Stable isotope ecology[M]. Beijing, China: Higher Education Press. ]

刘学炎, 肖化云, 刘丛强, 等. 2009. 基于石生藓类氮含量的 贵阳地区大气氮沉降 [J]. 生态学报, 29(12): 6646-6653. [Liu X Y, Xiao H Y, Liu C Q, et al. 2009. Quantification of atmospheric nitrogen deposition at Guiyang area based on nitrogen concentration of epilithicmosses[J]. Acta Ecologica Sinica, 29(12): 6646-6653. ]

娄焕杰, 邓焕广, 王东启, 等. 2013. 上海城市河岸带土壤反 硝化作用研究 [J]. 环境科学学报, 33(4): 1118-1126. [Lou H J, Deng H G, Wang D Q, et al. 2013. Soil denitrification in Shanghai urban riparian zone[J]. Acta Scienctiae Circumstantiae, 33(4): 1118-1126.]

秦树平, 胡春胜, 张玉铭, 等. 2011. 氮足迹研究进展 [J]. 中国 生态农业学报, 19(2): 462-467. [Qin S P, Hu C S, Zhang $\mathrm{Y}$ M, et al. 2011. Advances in nitrogen footprint research [J]. Chinese Journal of Eco-Agriculture, 19(2): 462-467.] 施亚岗, 崔胜辉, 许肃, 等. 2014. 需求视角的中国能源消费 氮氧化物排放研究 [J]. 环境科学学报, 34(10): 26842693. [Shi Y L, Cui S H, Xu S, et al. 2014. Nitrogen oxide emission in energy consumption in China from a consumption- based perspective[J]. Acta Scientiae Circumstantiae, 34(10): 2684-2693. ]

申卫国, 王辉, 李志, 等. 2010. 北京市交通道路空气 NOx 的 污染现状及时空变化规律研究 [J]. 环境工程学报, 4(5): 1139-1142. [Shen W G, Wang H, Li Z, et al. 2010. Study on the status and temporal and special variation of NOx pollution on urban traffic roads in Beijing[J]. Chinese Journal of Environmental Engineering, 4(5): 1139-1142. ]

遆超普, 颜晓元. 2010. 基于氮排放数据的中国大陆大气氮 素湿沉降量估算 [J]. 农业环境科学学报, 29(8): 16061611. [Ti C P, Yan X Y. 2010. Estimation of atmospheric nitrogen wet deposition in china mainland from based on emission data[J]. Journal of Agro- Environment Science, 29(8): 1606-1611.]

王德宣, 赵普生, 张玉霞, 等. 2010. 北京市区大气氮沉降研 究 [J]. 环境科学, 31(9): 1987-1992. [Wang D X, Zhao P S, Zhang Y X, et al. 2010. Atmospheric nitrogen deposition in urban area of Beijing[J]. Environmental Science, 31(9): 1987-1992. ]

王占山, 李云婷, 陈添, 等. 2015. 2013 年北京市 PM2.5 的时 空分布 [J]. 地理学报, 70(1): 110-120. [Wang Z S, Li Y T, Chen T, et al. 2015. Spatial- temporal characteristics of PM2.5 in Beijing in 2013[J]. Acta Geographica Sinica, 70 (1): 110-120. ]

魏静, 马林, 路光, 等. 2008. 城镇化对我国食物消费系统氮 素流动及循环利用的影响 [J]. 生态学报, 28(3):10161025. [Wei J, Ma L, Lu G, et al. 2008. The influence of urbanization on nitrogen flow and recycling utilization in food consumption system of China[J]. Acta Ecologica Sinica, 28(3): 1016-1025. ]

夏立忠, 杨林章, 吴春加, 等. 2003. 太湖地区典型小城镇降 水径流 NP负荷空间分布的研究 $[\mathrm{J}]$. 农业环境科学学 报, 22(3):267-270. [Xia L Z, Yang L Z, Wu C J, et al. 2003. Distribution of nitrogen and phosphorus loads in runoff in a representative town in Tai- Lake region[J]. 
Journal of Agro-Environment Science, 22(3): 267- 270. ]

冼超凡, 欧阳志云. 2014. 城市生态系统氮代谢研究进展 [J]. 生态学杂志, 33(9): 2548-2557. [Xian C F, Ouyang Z Y. 2014. Urban ecosystem nitrogen metabolism: research progress[J]. Chinese Journal of Ecology, 33(9): 25482557.]

徐志伟, 张心昱, 于贵瑞, 等. 2014. 中国水体硝酸盐氮氧双 稳定同位素溯源研究进展 [J]. 环境科学, 35(8): 32303238. [Xu Z W, Zhang X Y, Yu G R, et al. 2014. Review of dual stable isotope technique for nitrate source identification in surface-and Groundwater in China[J]. Environmental Science, 35(8): 3230-3238. ]

薛亦峰, 曲松, 间静, 等. 2014. 北京市水泥工业大气污染物 排放清单及污染特征 [J]. 环境科学与技术, 37(1): 201204. [Xue Y F, Qu S, Yan J, et al. 2014. Development of an air pollutant emission inventory of cement industry for Beijing and pollution characteristics analysis[J]. Environmental Science \&Technology, 37(1): 201-204. ]

袁玲, 周金斌, 辜夕容, 等. 2009. 重庆典型地区大气湿沉降 氮的时空变化 [J]. 生态学报, 129(11): 6095-6101. [Yuan L, Zhou X B, Gu X R, et al. 2009. Variation in wet deposition of nitrogen from atmosphere in typical areas of Chongqing[J]. Acta Ecologica Sinica, 129(11): 60956101. ]

余环, 王普才, 宗雪梅, 等. 2009. 奥运期间北京地区卫星监 测 $\mathrm{NO}_{2}$ 柱浓度的变化 [J]. 科学通报, 54(3): 299-304. [Yu H, Wang P C, Zhong X M, et al. 2009. The concentration changes of tropospheric $\mathrm{NO}_{2}$ column during the Olympic Games in Beijing $[\mathrm{J}]$. Chinese Science Bulletin, 54(3): 299-304. ]

于洋, 崔胜辉, 赵胜男, 等. 2012. 城市居民食物氮消费变化 及其环境负荷: 以厦门市为例 [J]. 生态学报, 32(19): 5953-5961. [Yu Y, Cui S H, Zhao S N, et al. 2012. Changes of residents nitrogen consumption and its environmental loading from food in Xiamen[J]. Acta Ecologica Sinica, 32(19): 5953-5961. ]

岳甫均, 李军, 刘小龙, 等. 2010. 利用氮同位素技术探讨天 津地表水氮污染 [J]. 生态学杂志, 29(7): 1403-1408. [Yue F J, Li J, Liu X L, et al. 2010. Nitrogen pollution of surface water in Tianjin based on nitrogen isotope approach[J]. Chinese Journal of Ecology, 29(7): 1403-1408.] 张强, 耿冠楠, 王斯文, 等. 2012. 卫星遥感观测中国 19962010 年氮氧化物排放变化 [J]. 科学通报, 57(16): 14461453. [Zhang Q, Geng G N, Wang S W, et al. 2012. Satellite remote sensing of changes in NOx emissions over China: 1996-2010[J]. Chinese Science Bulletin, 57(16): 1446-1453.

张柏发, 陈丁江. 2014. 1980-2010年浙江某典型河流硝态氮 通量对净人类活动氮输人的动态响应 $[\mathrm{J}]$. 环境科学, 35 (8): 2911-2919. [Zhang B F, Chen D J. 2014. Dynamic re- sponse of riverine nitrate flux to net anthropogenic nitrogen inputs in a typical river in Zhejiang Province over the 1980- 2010 period[J]. Environmental Science, 35(8): 2911-2919. ]

张汪寿, 李叙勇, 苏静君. 2014. 河流氮输出对流域人类活动 净氮输人的响应研究综述 [J]. 应用生态学报, 25(1):272278. [Zhang W S, Li X Y, Su J J. 2014. Responses of riverine nitrogen export to net anthropogenic nitrogen inputs: a review $[\mathrm{J}]$. Chinese Journal of Applied Ecology, 25(1): 272-278. ]

郑循华, 符聪斌, 徐星凯, 等. 2002. 亚洲氮循环案例研究 $[\mathrm{J}]$. Ambio, 31(2): 79-87. [Zheng X H, Fu C B, Xu X K, et al. 2002. The Asian nitrogen cycle case study[J]. AMBIO, 31 (2): 79-87. ]

朱兆良, 邢光喜. 2010. 氮循环: 敒关农业生产、环境保护与 人类健康(修订版) $[\mathrm{M}]$. 北京: 清华大学出版社. [Zhu Z L, Xing G X. 2010. Danxunhuan: youguan nongye shengchan , huanjing baohu yu renlei jiankan: xiuding ban [M]. Beijing, China: Tsinghua University Press]

Akimoto H, Ohara T, Kurokawa J, et al. 2006. Verification of energy consumption in China during 1996-2003 by using satellite observational data[J]. Atmospheric Environment, 40: 7663-7667.

Baker L A, Hope D, Xu Y, et al. 2001. Nitrogen balance for the Central Arizona-Phoenix(CAP) ecosystem[J]. Ecosystems, 4: 582-602.

Barles S. 2007. Feeding the city: food consumption and flow of nitrogen, Paris, 1801-1914[J]. Science of the Total Environment, 375: 48-58.

Beevers S D, Westmoreland E, Jong M C, et al. 2012. Trends in $\mathrm{NOx}$ and $\mathrm{NO}_{2}$ emissions from road traffic in Great Britain[J]. Atmospheric Environment, 54: 107-116.

Beirle S, Platt U, Wenig M, et al. 2003. Weekly cycle of $\mathrm{NO}_{2}$ by GOME measurements: a signature of anthropogenic sources[J]. Atmospheric Chemistry and Physics, 3: 2225 2232.

Bettez N D, Groffman P M. 2013. Nitrogen deposition in and near an urban ecosystem[J]. Environmental Science \& Technology, 47: 6047-6051.

Burian S J, Streit G E, McPherson T N, et al. 2001. Modeling the atmospheric deposition and stormwater washoff of nitrogen compounds[J]. Environmental Modelling \& Software, 16: 467-479.

Burns D A, Boyer E W, Elliott E M, et al. 2009. Sources and transformations of nitrate from streams draining varying land uses: evidence from dual isotope, analysis[J]. Journal of Environmental Quality, 38: 1149-1159.

Butler T J, Likens G E, Vermeylen F M, et al. 2005. The impact of changing nitrogen oxide emissions on wet and dry nitrogen deposition in the northeastern USA[J]. Atmo- 
spheric Environment, 39: 4851-4862.

Carslaw D C, Beevers S D, Fuller G. 2001. An empirical approach for the prediction of annual mean nitrogen dioxide concentrations in London[J]. Atmospheric Environment, 35: 1505-1515.

Chen F S, Fahey T J , Yu M Y, et al. 2010. Key nitrogen cycling processes in pine plantations along a short urban rural gradient in Nanchang, China[J]. Forest Ecology and Management, 259: 477-486.

Collins K A, Lawrence T J, Stander E K, et al. 2010. Opportunities and challenges for managing nitrogen in urban stormwater: a review and synthesis[J]. Ecological Engineering, 36: 1507-1519.

Cui S H, Shi Y L, Groffman P M, et al. 2013. Centennial-scale analysis of the creation and fate of reactive nitrogen in China (1910-2010)[J]. Proceedings of the National Academy of Sciences of the United States of America, 110(6): 2052-2057.

Cyrys J,Eeftens M, Heinrich J, et al. 2012. Variation of $\mathrm{NO}_{2}$ and NOx concentrations between and within 36 European study areas: results from the ESCAPE study[J]. Atmospheric Environment, 62: 374-390.

De Souza P A, Ponette-Gonz'alez A G, De Mello W Z, et al. 2015. Atmospheric organic and inorganic nitrogen inputs to coastal urban and montane Atlantic Forest sites in southeastern Brazil[J]. Atmospheric Research, doi: 10.1016/j.atmosres.2015.03.011.

Elliott E M, Kendall C, Wankel S D, et al. 2007. Nitrogen isotopes as indicators of NOx source contributions to atmospheric nitrate deposition across the midwestern and northeastern United States[J]. Environmental Science \& Technology, 41: 7661-7667.

Erisman J W. 2004. The Nanjing declaration on management of reactive nitrogen[J]. Bioscience, 54: 286-287.

Erisman J W, Grennfelt P, Sutton M. 2003. The European perspective on nitrogen emission and deposition[J]. Environment International, 29: 311-325.

Erisman J W, Galloway J N, Seitzinger S, et al. 2013. Consequences of human modification of the global nitrogen cycle[J]. Philosophical Transactions of the Royal Society of London Series B, Biological Sciences, 368: 1-9.

Felix J D, Elliott E M, Gish T, et al, 2014. Examining the transport of ammonia emissions across landscapes using nitrogen isotope ratios[J]. Atmospheric Environment, 95: 563570.

Fenn M E, Haeuber R, Tonnesen G S, et al. 2003. Nitrogen emissions, deposition, and monitoring in the Western United States[J]. BioScience, 53(4): 391-403.

Flint K R, Davis A P. 2007. Pollutant mass flushing characterization of highway storm-water runoff from an ultra-ur- ban area[J]. Journal of Environmental Engineering, 133: 616-626.

Forkes J. 2007. Nitrogen balance for the urban food metabolism of Toronto, Canada[J]. Resources, Conservation and Recycling, 52: 74-94.

Fowler D, Coyle M, Skiba U, et al. 2013. The global nitrogen cycle in the twenty-first century[J]. Philosophical Transactions of the Royal Society of London Series B, Biological Sciences, 368: 20130164. doi: 10. 1098/rstb. 2013 0164.

Galloway J N, Cowling E B. 2002. Reactive nitrogen and the world: 200 years of change[J]. AMBIO, 31(2): 64-71.

Galloway J N, Dentener F J, Capone D G, et al. 2004. Nitrogen cycles: past, present, and future $[\mathrm{J}]$. Biogeochemistry, 70: 153-226.

Galloway J N, Leach A M, Bleeker A, et al. 2013. A chronology of human understanding of the nitrogen cycle[J]. Philosophical Transactions of the Royal Society of London Series B, Biological sciences, 368: 20130120. doi: 10.1098/rstb. 2013. 0120 .

Galloway J N, Townsend A R, Erisman J W, et al. 2008. Transformation of the nitrogen cycle: recent trends, questions, and potential solutions[J]. Science, 320: 889-892.

Grimm N B, Faeth S H, Golubiewski N E, et al. 2008. Global change and the ecology of cities[J]. Science, 319: 756760

Grimm N B, Foster D, Groffman P, et al. 2008. The changing landscape: ecosystem responses to urbanization and pollution across climatic and societal gradients[J]. Frontiers in Ecology and the Environment, 6: 264-272.

Grimm N B, Grove J M, Pickett S T A, et al. 2000. Integrated approaches to long-term studies of urban ecological systems[J]. Bioscience, 50(7): 571-584.

Groffman P M, Law N L, Belt K T, et al. 2004. Nitrogen fluxes and retention in urban watershed ecosystems[J]. Ecosystems, 7: 393-403.

Gu B J, Chang J, Ge Y, et al. 2009. Anthropogenic modification of the nitrogen cycling within the Greater Hangzhou area system, China[J]. Ecological Applications, 19: 974 988.

Gu B J, Chang J, Min Y, et al. 2013. The role of industrial nitrogen in the global nitrogen biogeochemical cycle[J]. Scientific reports, doi: 10. 1038/srep02579.

Gu B J, Dong X L, Peng C H, et al. 2012. The long-term impact of urbanization on nitrogen patterns and dynamics in Shanghai, China[J]. Environmental Pollution, 171: 30-37.

Han Y, Li X, Nan Z. 2011. Net anthropogenic nitrogen accumulation in the Beijing metropolitan region[J]. Environmental Science and Pollution Research, 18: 485-496.

Hastings M G, Casciotti K L, Elliott E M. 2013. Stable iso- 
topes as tracers of anthropogenic nitrogen sources, deposition, and impacts[J]. Elements, 9: 339-344.

Howarth R W, Billen G, Swaney D, et al. 1996. Regional nitrogen budgets and riverine N\&P fluxes for the drainages to the North Atlantic Ocean: natural and human influences [J]. Biogeochemistry, 35(1): 75 - 139.

Hsieh C H, Davis A P, Needelman B A. 2007. Nitrogen removal from urban storm-water runoff through layered bio-retention columns[J]. Water Environment Research, 79(2): 177-184.

Jansson A, Johan C. 2007. Tradeoffs between environmental goals and urban development: the case of nitrogen load from the Stockholm County to the Baltic Sea[J]. AMBIO, 36(8): 612-618.

Jordan T E, Weller D E, Correll D L. 2003. Sources of nutrient inputs to the Patuxent River estuary[J]. Estuaries, 26: 226243.

Kaushal S S, Groffman P M, Band L E, et al. 2008. Interaction between urbanization and climate variability amplifies watershed nitrate export in Maryland[J]. Environmental Science \& Technology, 42: 5872-5878.

Kaye J P, Groffman P M, Grimm N B, Baker L A, et al. 2006. A distinct urban biogeochemistry[J]? Trends in Ecology and Evolution, 21: 192-199.

Keeney D R, Hatfield J L. 2001. The nitrogen cycle, historical perspective, and current and potential future concerns $[\mathrm{M}] / /$ Follett R F, Hatfield J L. Nitrogen in the environment: sources, problems and management. Amsterdam, the Netherlands and Boston, MA: Elsevier.

Kim H, Seagren E A, Davis A P. 2003. Engineered bio-retention for removal of nitrate from storm- water runoff[J]. Water Environment Research, 75(4): 355-367.

Lamsal L N, Martin R V, Parrish D D, et al. 2013. Scaling relationship for $\mathrm{NO}_{2}$ pollution and urban population size: a satellite perspective[J]. Environmental Science \& Technology, 47: 7855-7861.

Larson E K, Grimm N B. 2012. Small-scale and extensive hydro-geomorphic modification and water redistribution in a desert city and implications for regional nitrogen removal[J]. Urban Ecosyst, 15: 71-85.

Leach A M, Galloway J N, Bleeker A, et al. 2012. A nitrogen footprint model to help consumers understand their role in nitrogen losses to the environment[J]. Environmental Development, 1: 40-66.

Lin T, Gibson V, Cui S H ,et al. 2015. Managing urban nutrient biogeochemistry for sustainable urbanization[J]. Environmental Pollution, 192: 244-250.

Lohse K A, Hope D, Sponseller R, et al. 2008. Atmospheric deposition of carbon and nutrients across an arid metropoli$\tan \operatorname{area}[\mathrm{J}]$. Science of the Total Environment, 402: 95-105.
Luo Y Z, Yang X S, Carley R J, et al. 2003. Effects of geographical location and land use on atmospheric deposition of nitrogen in the state of Connecticut[J]. Environmental Pollution, 124: 437-448.

Ma L, Guo J H, Velthof G L, et al. 2014. Impacts of urban expansion on nitrogen and phosphorus flows in the food system of Beijing from 1978 to 2008[J]. Global Environmental Change, 28: 192-204.

Ma X, Wang Z, Yin Z, et al. 2008. Nitrogen flow analysis in Huizhou, South China[J]. Environmental Management, 41: 378-388

Ortiz-Zayas J R, Cuevas E, Mayol-Bracero O L, et al. 2006. Urban influences on the nitrogen cycle in Puerto Rico[J]. Biogeochemistry, 79: 109-133.

Paul M J, Meyer J L. 2001. Streams in the urban landscape[J]. Annual Review of Ecology and Systematics, 32: 333-365.

Pickett S T A, Cadenasso M L, Grove J M, et al. 2011. Urban ecological systems: scientific foundations and a decade of progress[J]. Journal of Environmental Management, 92: 331-362.

Ren Y F, Xu Z W, Zhang X Y, et al. 2014. Nitrogen pollution and source identification of urban ecosystem surface water in Beijing $[\mathrm{J}]$. Frontiers of Environmental Science \& Engineering, 8(1): 106-116.

Roberts A D, Prince S D. 2010. Effects of urban and non-urban land cover on nitrogen and phosphorus runoff to Chesapeake Bay[J]. Ecological Indicators, 10: 459-474.

Roberts A D, Prince S D, Jantz C A, et al. 2009. Effects of projected future urban land cover on nitrogen and phosphorus runoff to Chesapeake Bay[J]. Ecological Engineering, 35: 1758-1772.

Rojas A L P, Venegas L E. 2009. Atmospheric deposition of nitrogen emitted in the metropolitan area of Buenos Aires to coastal waters of De La Plata River[J]. Atmospheric Environment, 43: 1339-1348.

Russell A G, Winner D A, Harley R A, et al. 1993. Mathematical modeling and control of the dry deposition flux of nitrogen- containing air pollutants[J]. Environmental Science \& Technology, 27: 72-82.

Schlesinger W H. 2009. On the fate of anthropogenic nitrogen [J]. Proceedings of the National Academy of Sciences of the United States of America, 106(1): 203-208.

Svirejeva-Hopkins A, Reis S, Magid J, et al. 2011. Nitrogen flows and fate in urban landscapes[M]//Sutton M A, Howard C M, Erisman J W, et al. The European nitrogen assessment, sources, effects and policy perspectives. Cambridge, UK: Cambridge University Press: 249-270.

Sylvia C, Schaefer J T, Hollibaugh M A. 2009. Watershed nitrogen input and riverine export on the west coast of the USA[J]. Biogeochemistry, 93: 219-233. 
Townsend-small A, Pataki D E, Czimczik C I, et al. 2011. Nitrous oxide emissions and isotopic composition in urban and agricultural systems in southern California[J]. Journal of geophysical research, 116: 1-11. doi: 10. 1029/ 2010jg001494.

Vitousek P M, Aber J, Howarth R W, et al. 1997. Human alteration of the global nitrogen cycle: causes and consequences[J]. Ecological Applications, 7(3): 737-750.

Walsh C J, Fletcher T D, Ladson A R. 2009. Retention capacity: a metric to link stream ecology and stormwater management[J]. Journal of Hydrologic Engineering, 14: 399406.

Whitall D R, Paerl H W. 2001. Spatiotemporal variability of wet atmospheric nitrogen deposition to the Neuse River estuary, north Carolina[J]. Journal of Environmental Quality, 30(5): 1508-1515.
Wollheim W M, Pellerin B A, Vorosmarty C J, et al. 2005. N retention in urbanizing headwater catchments[J]. Ecosystems, 8: 871-884.

Xing G X, Zhu Z L. 2002. Regional nitrogen budgets for China and its major watersheds[J]. Biogeochemistry, 57-58: 405-427.

Yang X J. 2012. An assessment of landscape characteristics affecting estuarine nitrogen loading in an urban watershed [J]. Journal of Environmental Management, 94: 50-60.

Zhang Q, Streets D G, He K, et al. 2007. NO(x) emission trends for China, 1995-2004: the view from the ground and the view from space[J]. Journal of Geophysical Research, 112: D22306, doi: 10.1029/2007JD008684.

Zhu W X, Dillard N D, Grimm N B. 2004. Urban nitrogen biogeochemistry: status and processes in green retention basins $[\mathrm{J}]$. Biogeochemistry, 71: 177-196.

\title{
A review of urbanization impact on nitrogen cycle
}

\author{
GAO Qun', YU Cheng ${ }^{1,2}$ \\ (1. Key Laboratory of Watershed Geographic Sciences, Nanjing Institute of Geography and Limnology, CAS, \\ Nanjing 210008, China; 2. University of Chinese Academy of Sciences, Beijing 100049, China)
}

\begin{abstract}
Nitrogen $(\mathrm{N})$ cycle has been poorly characterized in urban ecosystems. In China, urbanization is an important process that alters urban ecosystem and urban nitrogen biogeochemical cycle. Human activities have more than doubled the nitrogen inputs to urbanized areas, and the sources, magnitude, spatiotemporal patterns, and drivers of $\mathrm{N}$ fluxes have been mediated by human activities. The imbalance of nitrogen cycle has far exceeded the safe limits of urban sustainable development in some urban areas in China. The urbanized areas are facing a series of environmental problems induced by excessive emission and deposition of reactive nitrogen. Urban smog is becoming very serious in a large number of Chinese cities. However, owing to the limited knowledge on the role of humans in the $\mathrm{N}$ biogeochemical cycle and the complexity and uncertainty of urban ecosystems, ecological research of nitrogen emission, deposition, transformation, removal, and retention in urban areas is relatively weak. Thus, comprehensively quantifying changes of nitrogen cycle in urbanized areas and understanding the effects of human activities, such as urbanization and economic development, on the variations of reactive nitrogen fluxes, have been a crucial topic in urban ecological research. This article reviews the state-of-the-art research in this field on the basis of comparison and analysis of Chinese and international studies. The problems exist in current research were summarized and future areas of research aiming at a much improved understanding of the scope of the anthropogenic N problem are discussed. With serious nitrogen pollution and environmental effects in urban areas, it is essential to carry out a long-term study of nitrogen flux and nitrogen budget to investigate the problem of nitrogen imbalance. It is necessary to think beyond the control of pollutant emissions and approach the issue and solution from an urban planning perspective and conduct comprehensive studies to understand the variation of nitrogen input and output trends and driving mechanisms.
\end{abstract}

Key words: urban smog; nitrogen cycle; nitrogen budget; urban ecosystem; urbanization 